\title{
Idiopathic Membranous Nephropathy at a Young Age
} \author{
Rashid Asghar ${ }^{1}$ and Sadia Majeed ${ }^{3}$ \\ ${ }^{1}$ Department of Nephrology, Multan institute of kidney diseases, Pakistan \\ ${ }^{2}$ Department of Medicine, Mayo Hospital, Lahore, Pakistan \\ ${ }^{3}$ Department of Pathology, Multan institute of kidney diseases, Pakistan
}

Muhammad Tanzeel Abbasi ${ }^{1 *}$, Mariam Arif ${ }^{2}$, Muhammad Nauman Hashmi ${ }^{1}$, Khuram Bashir ${ }^{1}$, Muhammad

*Corresponding author: Muhammad Tanzeel Abbasi, Consultant Nephrologist, Multan Institute of Kidney Diseases, Multan, Pakistan. Email: dr.tanzeel01@ hotmail.com

ORCID Id: 0000-0003-3939-3182

Received Date: April 02, 2021

Published Date: May 07, 2021

\begin{abstract}
Primary or idiopathic membranous nephropathy (MN) is distinctive and one of the most common causes of nephrotic syndrome in adults. It is quite prevalent in all races and has no gender predisposition. However, white adults are at more risk of MN. It is a relatively less common entity in pediatric population. We report a case of 12 years old female with idiopathic MN. She had no symptoms suggestive of nephrotic syndrome and was normotensive. She had incidental finding of proteinuria which was investigated, and renal biopsy revealed the features of MN.
\end{abstract}

Keywords: Primary MN; Membranous GN in children

\section{Introduction}

Membranous nephropathy (MN) is one of the common causes of idiopathic nephrotic syndrome. MN can be primary (idiopathic) or secondary. In young females, MN is usually secondary to systemic lupus erythematosis (SLE). It is seen in all racial and ethnic groups however it is more common in white males above 40 years of age. One third of the renal biopsies of non-diabetic adult patients turn out to be membranous nephropathy.

\section{Case}

This case report is about a 12 years old female who had incidental finding of proteinuria in her routine urine analysis. She had no complains of peri-orbital puffiness, ankle edema or urine frothiness. Her renal functions were normal. Ultrasound abdomen revealed normal sized kidneys. Routine urine analysis had proteins $(++)$, Blood (++) and RBC's (20-22/HPF). Her 24 hours urine analysis showed proteinuria of $914 \mathrm{mg} / 24$ hours. Her ANA and antidsDNA levels were normal.

In view of active urine and approximately 1 gram per 24 hours proteinuria, she underwent kidney biopsy. On light microscopy, all glomeruli showed diffuse thickening of basement membranes on periodic acid Schiff (PAS) stain (Figure 1) and spikes on silver stain (Figure 2). Immunofluorescence findings showed predominant IgG and C3 deposits (Figures 3\&4). Her anti phospholipase A2 receptor (anti PLA2R) antibodies were done which were positive with a titer of $63 \mathrm{RU} / \mathrm{ml}$ (>20 $\mathrm{RU} / \mathrm{ml}$ is positive).

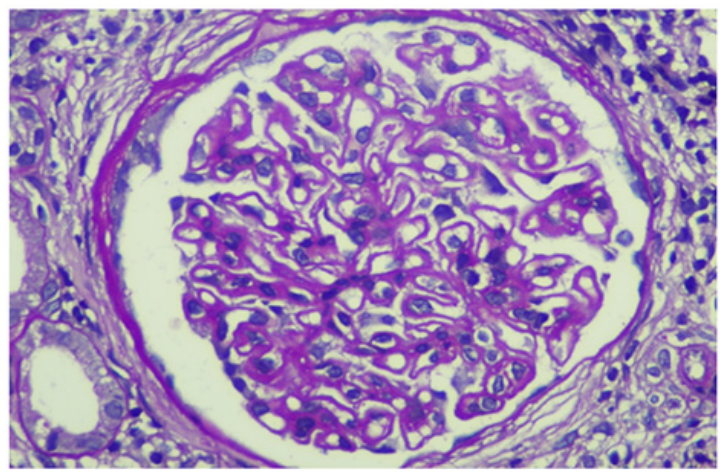

Figure 1: Diffuse thickening of glomerular basement membrane on PAS stain. 


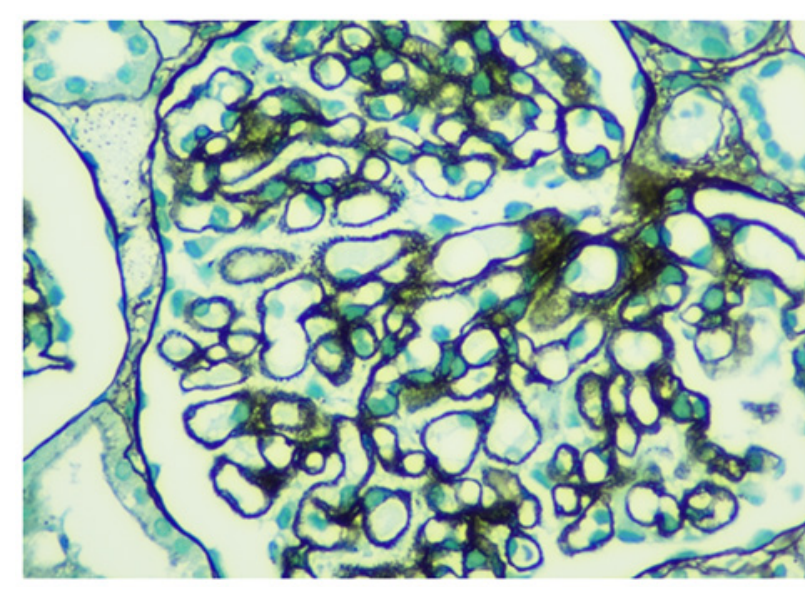

Figure 2: Spikes on silver stain.

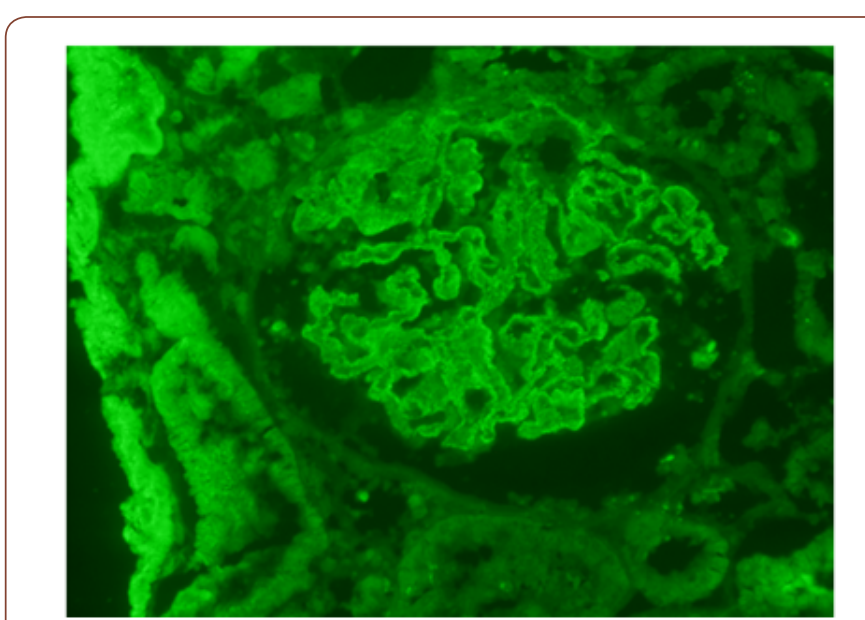

Figure 3: IF shows predominant IgG deposits.

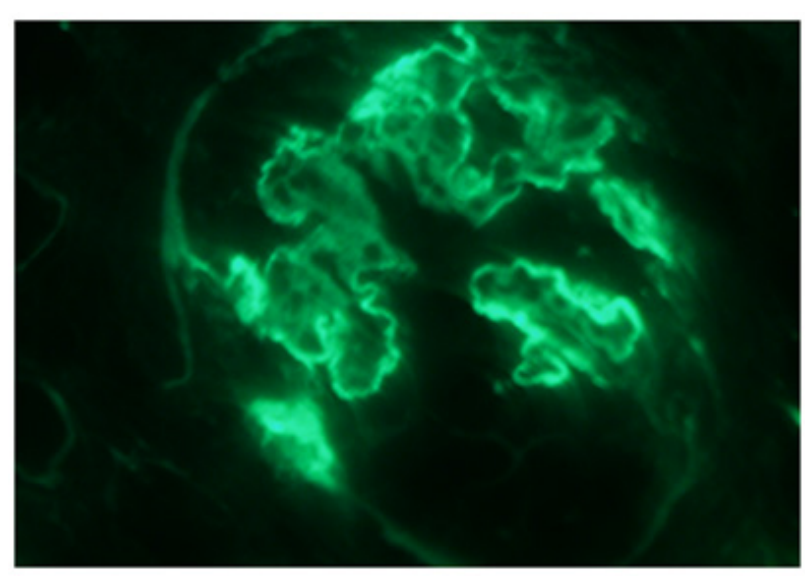

Figure 4: C3 deposits on IF.

\section{Discussion}

$\mathrm{MN}$ is among one of the common causes of nephrotic syndrome in adults [1]. It is seen in all racial and ethnic groups. However, it is more prevalent in white adults. There is no gender predisposition. About $80 \%$ cases of MN are renal-limited (primary or idiopathic) whereas $20 \%$ cases are secondary which can be because of systemic lupus erythematosis, infections like hepatitis B, solid organ tumors, drugs or toxins [2]. It is more prevalent after 4th decade of life [3].

In children, nephrotic syndrome is a rare entity and is usually due to minimal change disease [4]. Only $1-7 \%$ of the renal biopsies done in pediatric population show features of MN. Patients usually present with hematuria, proteinuria and edema but atypical presentation may be there [5]. The characteristic changes on renal biopsy is diffuse glomerular basement membrane (GBM) thickening and spikes on light microscopy (LM) [6]. In early stages of MN, there is effacement of podocyte foot processes and homogenous immune deposits in sub epithelial distribution. These immune deposits give the appearance of spikes on LM. This basement membrane material is later on laid between these deposits leading to formation of new thickened basement membrane. Immunofluorescence (IF) findings include diffuse granular staining of IgG and C3 along GBM. Subepithelial deposits and podocyte effacement are seen along GBM on electron microscopy.

Usually the patients respond well to conservative management with angiotensin converting enzyme inhibitors (ACE-i) or angiotensin receptor blockers (ARB`s). In a study, Ramirez, et al reported an increased risk of progression to renal insufficiency in children with MN [7]. However, in another study by Latham et al found no correlation between initial histopathological findings and clinical outcome [8]. Obana et al revealed two patterns of MN, segmental and diffuse glomerular however, histological findings were not evaluated as prognostic tool in this study [9].

LM findings are same in primary and secondary MN. In secondary $\mathrm{MN}$, there is full house pattern (all immunoglobulin including C3, C4 and C1q) on IF but this pattern is not seen in idiopathic MN. C1q has a strong association with secondary MN. Moreover, Anti PLA2R antibodies are present in in 70-80\% cases of primary MN [10]. The response to treatment is strongly associated with baseline Anti PLA2R levels. Low baseline Anti PLA2R titers are associated with improvement in proteinuria with treatment. Light microscopy and immunofluorescence findings along with positive anti PLA2R antibodies and negative ANA and anti dsDNA confirmed the diagnosis of Primary MN in our case.

\section{Conclusion}

Primary MN is one of the common nephrotic syndrome in adults but it is rarely seen in pediatric population. Progression of this diseases in pediatric population is rare. In children, this diseases behaves less aggressively and immunosuppression is usually not required. In our case, patient was reassured and conservative management was advised. She is in remission at present with conservative management and under nephrology follow up. 


\section{Acknowledgement}

\section{None}

\section{Ethics Committee Approval}

Our manuscript does not report on or involve the use of any animal or human data or tissue, and not contain any individual persons' data, so this section is not applicable to our submission.

\section{Informed Consent}

Informed oral consent was taken from patient and her father

\section{Conflict of Interest}

None.

\section{Financial Disclosure}

No grants or funding.

\section{References}

1. Couser WG (2017) Primary Membranous Nephropathy. Clin J Am Soc Nephrol 12 (6): 983-997.

2. Kaplan BS, Meyers KE (2004) Pediatr Nephrol Urol: 179-181.
3. Ayalon R, Beck LH Jr (2015) Membranous nephropathy: not just a disease for adults. Pediatr Nephrol 30(1):31-39.

4. Cameron JS (1990) Membranous nephropathy in childhood and its treatment. Pediatr Nephrol 4(2):193-198.

5. Avner ED, Harmon WE, Niaudet P (2004) Pediatric Nephrology: 643645.

6. Wasserstein AG (1997) Membranous glomerulonephritis. J Am Soc Nephrol 8(4): 664-674.

7. Ramirez F, Brouhard BH, Travis LB, Ellis EN (1982) Idiopathic membranous nephropathy in children. J Pediatr 101(5): 677-681.

8. Latham P, Poucell S, Koresaar A, Arbus G, Baumal R (1982) Idiopathic membranous glomerulopathy in Canadian children: a clinicopathologic study. J Pediatr 101(5): 682-685.

9. Obana M, Nakanishi K, Sako M, Yata N, Nozu K, et al. (2006) Segmental Membranous Glomerulonephritis in Children: Comparison with Global Membranous Glomerulonephritis. Clin J Am Soc Nephrol 1(4): 723-729.

10. Beck LH Jr, Bonegio RG, Lambeau G, Beck DM, Powell DW, et al. (2009) M-type phospholipase A2 receptor as target antigen in idiopathic membranous nephropathy. N Engl J Med 361(1): 11-21. 